\section{Cadherin keeps order}

Cadherins are adhesion molecules with important roles in CNS development. Köster and colleagues now report in PLoS Biology that $\mathrm{N}$-cadherin (also known as cadherin 2) is required for maintaining cell-cell contacts and cell polarity in granule cells (GCs) during directional migration.

Time-lapse confocal imaging of zebrafish embryos stably expressing

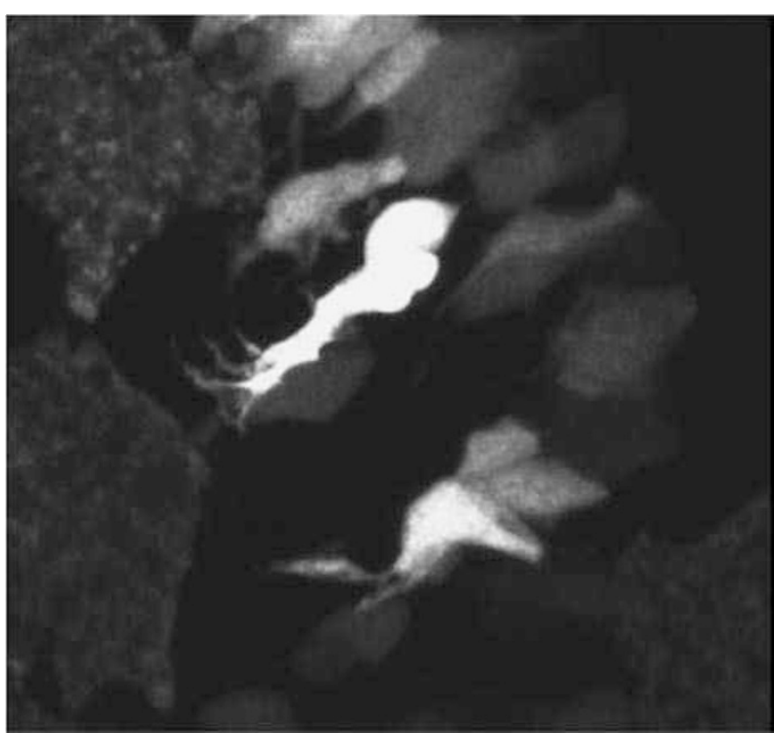

In vivo image of cerebellar granule cells leaving the rhombic lip in groups to form chain-like structures. Image courtesy of R. Köster, German Research Centre for Environmental Health, Germany. green fluorescent protein (GFP) in cerebellar GCs showed that the cells move from the upper rhombic lip towards the midbrain-hindbrain boundary by forming chain-like structures. Transmission electron microscopy revealed that adherens junction-mediated cell-cell contacts are important for cohesive movement.

The authors found that on initiation of migration GCs form distinct clusters that eventually differentiate to form the GC compartments of the cerebellum. By contrast, GCs lacking $\mathrm{N}$-cadherin failed to form clusters or migrate. Injecting these mutant GCs with wild-type $\mathrm{N}$-cadherin mRNA restored migration; however, the cells failed to form migratory chains and reach their target. Time-lapse imaging of mutant embryos with transplanted wild-type cells showed that mutant cells can only form migratory chains with the transplanted $\mathrm{N}$-cadherin-expressing wild-type GCs, suggesting that $\mathrm{N}$-cadherin acts in an autonomous manner to mediate cohesive directional migration of GCs.

GCs migrate through nucleokinesis - the forward transport of the nucleus along microtubules, which requires the centrosome to be positioned in front of the nucleus and towards the leading edge of the cell. Using fluorescent labels, the authors demonstrated that the centrosome was positioned at the leading edge in most wild-type GCs, whereas its position varied between the cell front and rear in GCs lacking N-cadherin. Time-lapse imaging revealed that $\mathrm{N}$-cadherin dynamically relocalizes from the cell rear in resting cells towards the cell front in elongating and migrating cells to stabilize the centrosome. Taken together, these results indicate that $\mathrm{N}$-cadherin has a key role in the directional migration of cerebellar GCs by coordinating cell-cell adhesion and cell polarity.

ORIGINAL RESEARCH PAPER Rieger, S., Senghaas, N., Walch, A. \& Köster, R. W.

Cadherin-2 controls directional chain migration of cerebellar granule neurons. PLoS Biol. 7, e1000240 (2009)

FURTHER READING Takeichi, M. The cadherin superfamily in neuronal connections and interactions. Nature Rev. Neurosci. 8, 11-20 (2007)| Chausovsky, A., Bershadsky, A. D. \& Borisy, G. G. Cadherin-mediated regulation of microtubule dynamics. Nature Cell Biol. 2, 797-804 (2000)| Leah, E. Cell migration: cadherin glycans branch out. Funct. Glycomics 12 Nov 2009 (doi:10.1038/ fg.2009.34)|Cell Migration Gateway: http:// www.cellmigration.org/index.shtml 